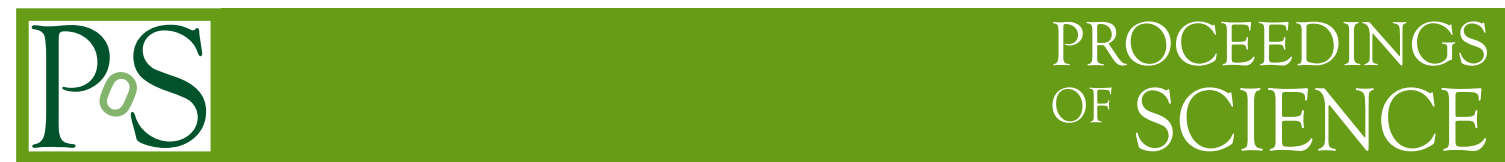

\title{
Finite-temperature QCD
}

\author{
M. Laine* \\ Faculty of Physics, University of Bielefeld, D-33501 Bielefeld, Germany \\ E-mail: laine@physik.uni-bielefeld.de
}

I start by discussing recent ideas concerning three different heavy quark related observables in finite-temperature QCD. Subsequently selected studies related to light quarks and gluons are reviewed, with a focus on thermodynamic observables, screening masses, and sum rules.

The XXVII International Symposium on Lattice Field Theory - LAT2009

July 26-31 2009

Peking University, Beijing, China

${ }^{*}$ Speaker. 


\section{Introduction}

The finite-temperature plenary talks at the yearly Lattice conferences have traditionally been overviews of results obtained during the past year or so [1]-[3]. This time, using an "outsider" status as an excuse, I would like to depart from the tradition and start with a somewhat more "active" approach, outlining a few newer ideas with the hope that this may help to inspire future work (sec. 2). Subsequently, however, I return to time-honoured practices and summarize results that caught my attention during recent months (sec. 3).

More precisely, the basic novelty that I would like to elaborate on is the slight paradigm shift that has been taking place concerning the role that heavy quarks (charm and bottom quarks) may play in hot QCD (with a temperature $T \sim 150-500 \mathrm{MeV}$ ). Given that the masses of the heavy quarks are much above the temperature, by up to an order of magnitude, it was long thought that they would be relatively "inert" and play little role in this temperature range. It has been one of the remarkable empirical discoveries of the Relativistic Heavy Ion Collider (RHIC) at Brookhaven, though, that even within the short lifetime of the thermal system the heavy quarks do appear to experience significant interactions with it. In fact, this is one of the reasons why the medium generated in heavy ion collisions is nowadays conceived to be a "strongly coupled" one.

Before embarking on a specific discussion on this topic, let me try to place my presentation in a wider context. Indeed, high-temperature $\mathrm{QCD}$, or pure $\mathrm{SU}\left(N_{\mathrm{c}}\right)$ gauge theory, can be pursued to many different goals. These theories offer, for instance, a tractable theoretical limit in which to study various aspects of confinement and chiral symmetry breaking, and several recent papers as well as parallel and poster contributions were formulated in this spirit (see, e.g., refs. [4]). On the phenomenological side, the original motivation for considering hot QCD was the possible role that it may play in Early Universe cosmology, and indeed the relic density of certain dark matter candidates is sensitive to the QCD equation-of-state (see, e.g., refs. [5]). Currently the most pressing issue of the field is, however, to offer QCD-based non-perturbative predictions for the observables that play a role in on-going and future heavy ion collision experiments, so this will be the focus of the present talk.

\section{Heavy quarks at high temperature}

Traditionally it was assumed, based on leading-order weak-coupling computations, that charm quarks would not have time to thermalize in heavy ion collisions, neither "kinetically" nor "chemically". Kinetic thermalization means that the momenta of the heavy quarks be distributed thermally, i.e. that the average momentum vanish in the rest frame of the thermal system and the average momentum-squared be proportional to the temperature. Chemical thermalization means that the number density (or, more properly, the entropy density) associated with the heavy quarks be as large as thermal field theory predicts.

A kind of a paradigm shift, associated with the concept of a "strongly coupled quark-gluon plasma", has however been taking place during the last few years. Indeed, experimental observations (to be reviewed below) concerning the "quenching" of heavy quark jets can be interpreted as indirect evidence for their kinetic thermalization; that is, heavy quarks interact more strongly than originally expected. Whether a chemical equilibration also takes place is not quite as obvious; 
changes in number density are accompanied by an additional Boltzmann factor, $\exp (-2 M / T) \ll 1$, where $M$ is a heavy quark mass and the factor two accounts for the fact that quarks and antiquarks come in pairs. On the other hand, the initial state already contains a distribution of heavy quarks and antiquarks originating from the very first hard scatterings, and this distribution may happen to be of the right order of magnitude; if so, only kinetic thermalization is required in order to bring them to full equilibrium. In any case it is now a challenge for theorists to, first of all, understand quantitatively the rapid kinetic thermalization of heavy quarks, and second, just in case, to probe how big an effect chemically thermalized charm quarks would have in the hydrodynamic modelling of heavy ion collision experiments.

In order to organize the corresponding discussion, I start by considering the case of " 0 valence" heavy quarks; by this I refer to the effect of (chemically thermalized) heavy sea quarks on the equation of state. This case can also be identified as QCD with $N_{\mathrm{f}}=2+1+1$ flavours. I then proceed to the " 1 valence" sector, considering heavy quark jets and their kinetic thermalization; and end with " $1+\overline{1}$ valence" heavy quarks, meaning heavy quarkonium. As we will see, there is a perspective for progress on all these fronts.

Before proceeding, I would like to briefly ponder the question of when it is precisely that some quark is "heavy", compared with the temperature $T$. To this effect, recall the form of the free quark propagator in continuum:

$$
\langle\psi(P) \bar{\psi}(Q)\rangle_{0}=\delta(P-Q) \frac{-i P+M}{P^{2}+M^{2}}, \quad P=\left(\omega_{n}, \mathbf{p}\right),
$$

with $\omega_{n}= \pm \pi T, \pm 3 \pi T, \ldots$. We see that the relevant comparison is something like $M \leftrightarrow \pi T$, but a question remains whether, once interactions are taken into account, we should insert the $\overline{\mathrm{MS}}$ mass, say $M_{c}^{\overline{\mathrm{MS}}}(3 \mathrm{GeV}) \approx 1 \mathrm{GeV}$; the pole mass, say $M_{c}^{\text {pole }} \sim(1.5-2.0) \mathrm{GeV}$; or something else. There is probably no unique answer to this question; rather, the answer depends on the observable. In any case, the message to take home is that in principle charm quarks could be "light" as soon as $T>1 \mathrm{GeV} / \pi \sim 300 \mathrm{MeV}$, or "heavy" as long as $T<2 \mathrm{GeV} / \pi \sim 600 \mathrm{MeV}$.

\section{1 "0 valence" - charm quark effect on $(e-3 p) / T^{4}$}

It was suggested a few years ago, based on a next-to-leading order weak-coupling analysis, that charm quarks may have a significant effect on various thermodynamic observables at surprisingly low temperatures [6]. The issue then is whether lattice studies could consolidate this suggestion.

As far as the lattice goes, it is appropriate to point out that although we conceptually now consider QCD with $N_{\mathrm{f}}=2+1+1$ flavours, with charm quarks in the thermal sea, the lattice estimate of their effect can in practice be reduced to the measurement of certain condensates, which can then be evaluated within the $2+1$ flavour theory. In this approach the charm quarks are only partly dynamical. Nevertheless, the order of magnitude of their effect should still come out right.

More concretely, focussing on the trace of the energy-momentum tensor, i.e. $(e-3 p) / T^{4}$, the heavy quark contribution can be reduced to [7]

$$
\Delta\left(\frac{e-3 p}{T^{4}}\right)=Z_{1} \times\left(\langle\bar{\psi} \psi\rangle_{T}-\langle\bar{\psi} \psi\rangle_{0}\right),
$$

where $Z_{1} \propto \partial M_{\mathrm{bare}} / \partial \beta_{\mathrm{L}}$ is a coefficient function that needs to be determined along the lines of constant physics ( $\beta_{\mathrm{L}}$ refers to the lattice $\beta$-coefficient). Recent measurements, from asqtad fermions 

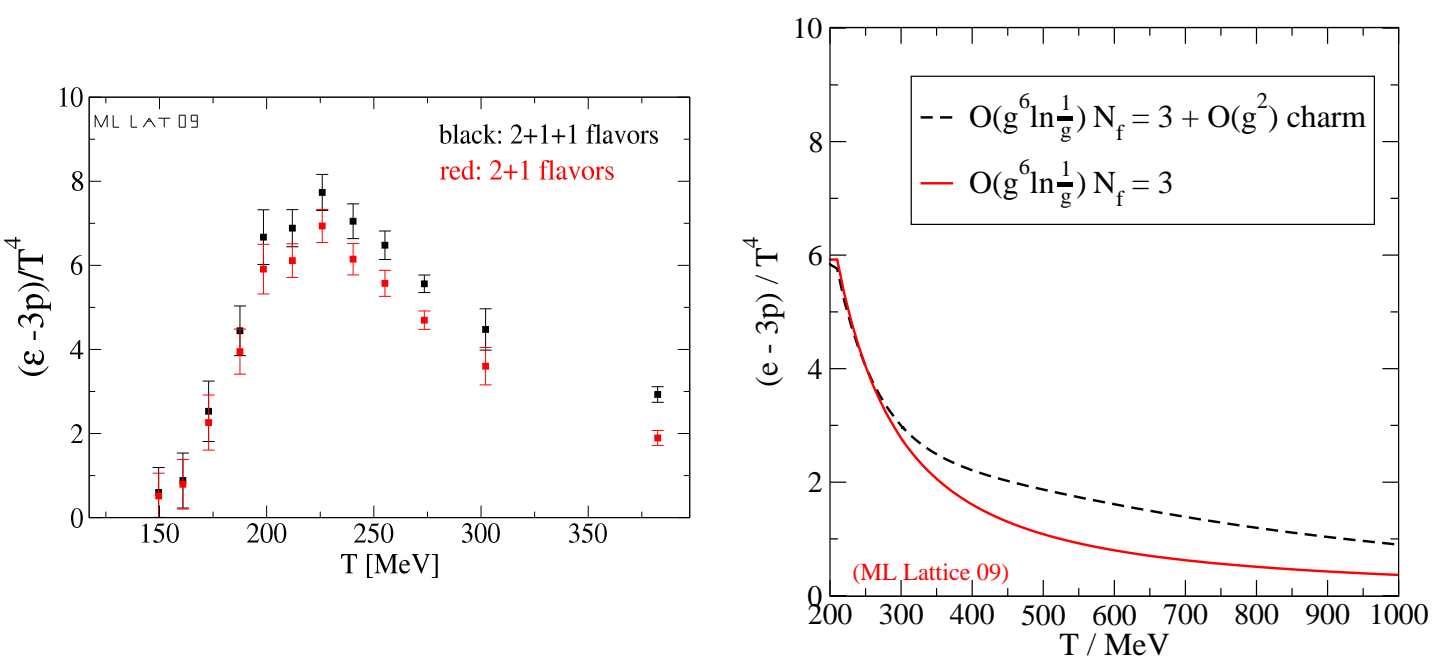

Figure 1: The charm quark contribution to the trace anomaly according to recent lattice simulations (left) [8], as well as resummed perturbation theory (right) [6].

with $N_{\tau}=6$ and bare mass ratios $M_{\text {bare }} / m_{s} \sim m_{s} / m_{u} \sim 10$ [8], are compared with the corresponding curve from perturbation theory in fig. 1 .

We observe that while the effect of charm quarks is very small at $T \lesssim 250 \mathrm{MeV}$, their relative importance in the trace anomaly increases rapidly with temperature, reaching $\gtrsim 50 \%$ already at $T \gtrsim 400 \mathrm{MeV}$. (The relative effect is somewhat smaller in quantities like the pressure or energy density, where no subtraction is carried out.)

To summarize, if chemical equilibration takes place, then charm quarks might affect the initial stages of hydrodynamics in future heavy ion collisions at the LHC, where higher temperatures may be reached than at the RHIC. In any case, the charm quarks do play a significant role in the equation of state relevant for cosmology, in which environment chemical equilibrium is guaranteed to be reached for all strongly interacting particles.

\section{2 "1 valence" - heavy quark jets}

In any high energy collision, a number of heavy quarks (and antiquarks) are produced in an initial hard process, as can be illustrated by the following Feynman diagram:

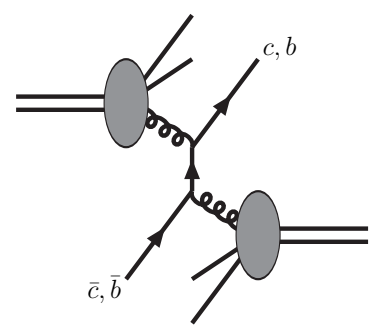

Assuming a suitable factorized framework and making use of previous experience gathered in $p+\bar{p}$ or $p+p$ collisions, it is believed that the corresponding production cross section is relatively well understood also for collisions involving heavy nuclei, such as $d+A u$ and $A u+A u$ [9]. Subsequent to their production, the heavy quarks decay, often semi-leptonically as $c \rightarrow \ell v X$. The leptons $\ell$ can 


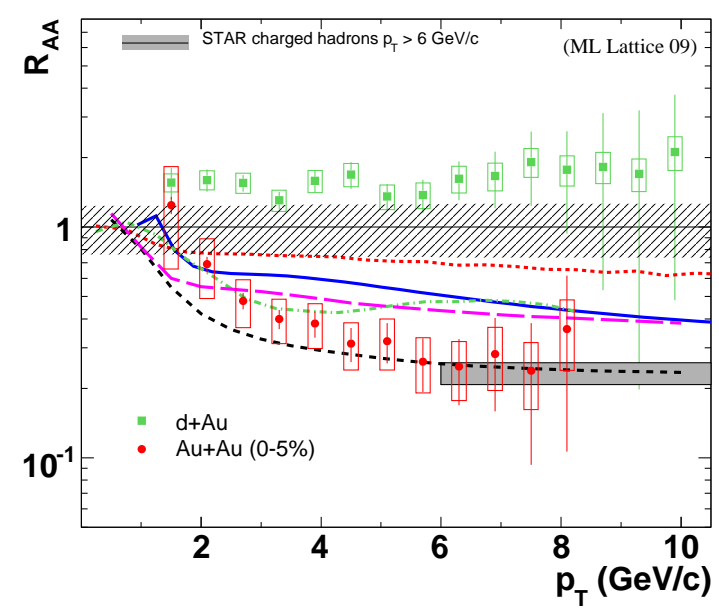

Figure 2: Experimental results from ref. [10] (see also ref. [11]), indicating that heavy quark jets propagate as expected in $d+A u$ collisions, but get quenched in $A u+A u$ collisions.

be observed, and the outcome can then be compared with the theoretical prediction.

It turns out that while results from $d+A u$ collisions indeed conform with theoretical expectations, those from $A u+A u$ collisions appear not to do so; rather, less leptons $\ell$ are observed than expected. The results are illustrated in fig. 2 in terms of the so-called "nuclear modification factor", $R_{A A}$, which is significantly below unity for $A u+A u$ collisions [10]; we can say that heavy quark jets get "quenched". Another relevant observable is the so-called elliptic flow, and the indication is that heavy quarks do participate in this hydrodynamic behaviour [11]. Both observations point towards the interpretation that, due to multiple scatterings with other particles, the heavy quarks slow down with respect to the thermal medium, and then flow together with it. I refer to this phenomenon as kinetic thermalization. In fact, the heavy quarks behave much like heavy particles in classical non-relativistic Brownian motion, and many of the same concepts can be argued to apply [12].

Let us denote the rate at which heavy quarks, assumed already to be close to rest, slow down, by $\eta_{D}$; this thermalization rate is often also called the "drag coefficient". Inspired by the analogy with Brownian motion and its classical description through Langevin dynamics, $\eta_{D}$ can be fluctuation-dissipation-related to another coefficient, $\kappa$, characterizing the autocorrelation of the force that acts on the heavy quarks $[12,13]$ :

$$
\eta_{D}=\frac{\kappa}{2 M_{\mathrm{kin}} T}\left(1+O\left(\frac{\alpha_{s}^{3 / 2} T}{M_{\mathrm{kin}}}\right)\right), \quad \kappa=\lim _{\omega \rightarrow 0} \frac{2 T \rho_{E}(\omega)}{\omega},
$$

where $M_{\text {kin }}$ refers to a particular heavy quark mass definition, related to the pole mass at zero temperature. The function $\rho_{E}$ is the spectral function corresponding to the Euclidean correlator (assuming $T>T_{\mathrm{c}}$ or $N_{\mathrm{f}}>0$ ) [13]

$$
G_{E}(\tau)=-\frac{1}{3} \sum_{i=1}^{3} \frac{\left\langle\operatorname{Re} \operatorname{Tr}\left[U_{\beta ; \tau} g E_{i}(\tau, \mathbf{0}) U_{\tau ; 0} g E_{i}(0, \mathbf{0})\right]\right\rangle}{\left\langle\operatorname{Re} \operatorname{Tr}\left[U_{\beta ; 0}\right]\right\rangle},
$$




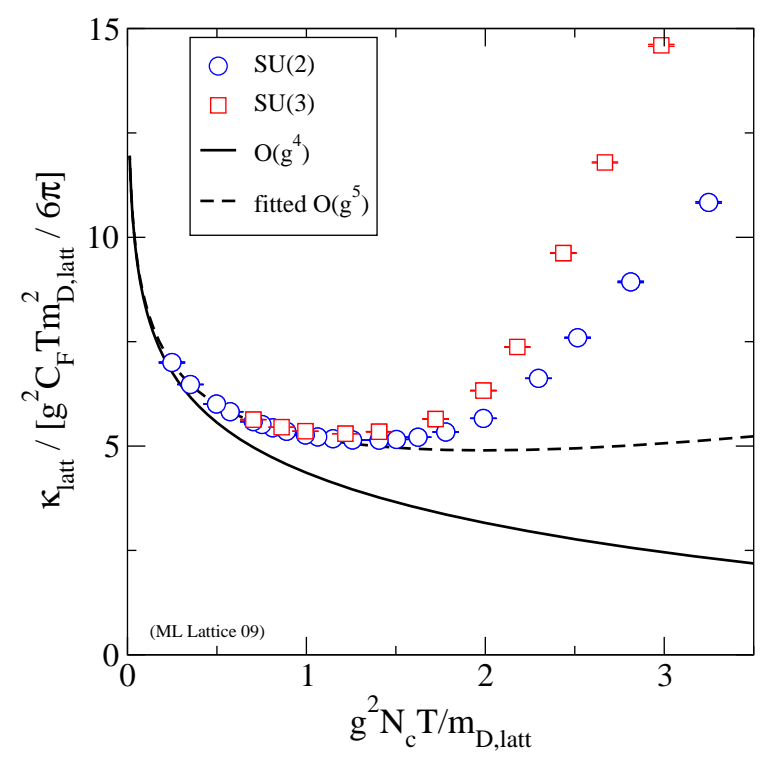

Figure 3: Results for the force-force transport coefficient $\kappa$ within classical lattice gauge theory, plotted as a function of the perturbative expansion parameter $g^{2} N_{\mathrm{c}} T / m_{\mathrm{D} \text {, latt }}$ (from ref. [14]). The non-perturbative data can exceed the leading-order and even the next-to-leading order results by a significant amount.

where $g E_{i} \equiv i\left[D_{0}, D_{i}\right]$ is the colour-electric field (shown here in continuum notation), and $U_{\tau_{\mathrm{b}}, \tau_{\mathrm{a}}}$ is a Wilson line in the Euclidean time direction, from $\tau_{\mathrm{a}}$ to $\tau_{\mathrm{b}}$. The numerator can be illustrated as

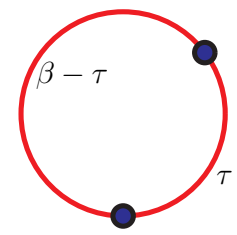

where the circle represents the Polyakov loop around the Euclidean time direction and the blobs denote electric field insertions. As far as I know no lattice measurements of this correlator have been published yet, although they should not be overwhelmingly demanding.

Even though no actual measurements are available, another test has been carried out. Indeed, the electric field correlator can also be addressed within "classical lattice gauge theory", which serves as kind of an effective low-energy description of infrared phenomena at finite temperatures [15]. It turns out that classical lattice gauge theory is more sensitive to ultraviolet physics than would be expected from a proper effective field theory framework; nevertheless, the result is interesting if plotted in terms of a quantity that does have a direct analogue in QCD, the so-called Debye mass parameter, $m_{\mathrm{D} \text {,latt }}^{2} \sim g^{2} T / a$. A result is shown in fig. 3 , including comparisons with a leading order perturbative result and a fitted next-to-leading order behaviour (the next-to-leading order result has been computed analytically in continuum QCD [16], but not in classical lattice gauge theory, where only its parametric form is known).

It can now be observed that if we insert the estimate $g^{2} T \sim m_{\mathrm{D} \text {, latt }}$, which is known to be a reasonable one on the QCD side, then we are in a regime where the next-to-leading order correction is of order $100 \%$, yet the non-perturbative result is larger still. Excitingly, such a significant increase appears to be more or less on top of what is phenomenologically needed in order to explain the fast 


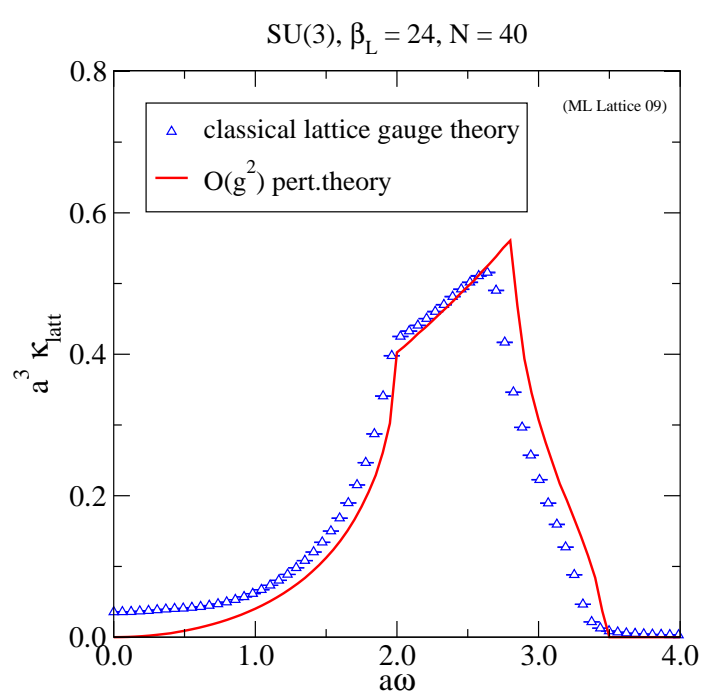

Figure 4: The combination $2 T \rho_{E}(\omega) / \omega$ from eq. (2.3), in lattice units, according to classical lattice gauge theory (from ref. [14]). The intercept at $\omega=0$ yields the result plotted in fig. 3 in different units.

thermalization (quenching) of heavy quark jets (see, e.g., ref. [17] and references therein).

Classical lattice gauge theory makes another prediction as well. In fig. 4, the frequencydependent function from which the intercept is to be taken according to eq. (2.3), is shown. The basic observation is that this function is flat at small frequencies; it has no transport peak, unlike spectral functions related to conserved currents. A similar flat behaviour for the spectral function related to the electric field correlator has also been observed in a very different theory, strongly coupled $\mathscr{N}=4$ Super-Yang-Mills theory in the large- $N_{\mathrm{c}}$ limit, handled through its AdS/CFT dual [18].

All this suggests that the electric field correlator might be more amenable to analytic continuation than current-current correlators from which viscosities and conductivities have been extracted previously [19]. To summarize, I can only solicit numerical tests of eq. (2.4), and hope that the outcome might yield a large coefficient $\kappa$, differing from the perturbative one by as much as an order of magnitude. With the advent of LHC, which should produce data on bottom quark jets as well, containing a different $M_{\text {kin }}$ in eq. (2.3) than in the charm quark case, our understanding of heavy quark jets within a hot medium could then be quantitatively tested.

\section{3 " $1+\overline{1}$ valence" - heavy quarkonium}

As a last example of heavy quark related observables, I briefly summarize recent news from heavy quarkonium physics. Like heavy quarks, heavy quarkonium can originally be generated in a non-thermal hard scattering, or through a thermal fluctuation. The latter process is reminiscent of those contributing to the chemical thermalization of single heavy quarks, and therefore probably too slow to take place effectively; nevertheless, surprises cannot be excluded. After having formed one way or the other, heavy quarkonium propagates through the thermal medium, whereby its properties get modified; therefore the quarkonium peak observed in the dilepton rate [20] may change in magnitude, shape, or position, depending on the temperature that is reached in the collision [21]. Some relevant Feynman diagrams are illustrated in fig. 5. 

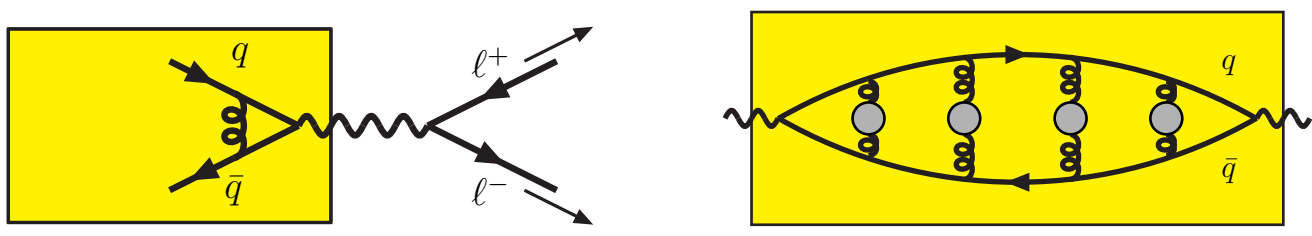

Figure 5: Left: an amplitude corresponding to the production of a dilepton pair from a thermalized medium. Right: squaring the amplitude, the dilepton production rate is seen to be proportional to the two-point correlation function of the electromagnetic current, with significant near-threshold corrections coming from medium-modified Coulomb exchange.

Now, like always in quarkonium physics, it appears reasonable to try and address the thermal modification of quarkonium properties through a potential model, thereby resumming corrections from graphs of the type in fig. 5(right). At finite temperatures, however, this is complicated by the multitude of different potentials that can in principle be defined, and intuitive arguments alone cannot decide which of them is the correct one. What is needed is rather a derivation of the relevant effective framework from QCD; at least within perturbation theory this can indeed be achieved [22], resulting in a potential-model type recipe for computing the spectral function [23], with a definite (in general complex) potential appearing as a "matching coefficient".

In order to appreciate the intricacies of the issue, it is important to realize that, on a Euclidean lattice, the time extent $\beta=1 / T$ is in some sense always "small"; more quantitatively, it can be argued that quarkonium melts at a temperature where parametrically $\beta<1 / \alpha_{s} M_{\text {kin }}$ [23]. In contrast, the Minkowskian time scale $t$ corresponding to the Coulombian binding energy of heavy quarkonium is "large", $t \sim 1 / \alpha_{s}^{2} M_{\text {kin. }}$. So, in the heavy quark limit where the effective $\alpha_{s}$ is small, we see that $t \gg \beta$, and it is more or less clear that the potential relevant for discussing quarkonium binding and dissociation at finite temperatures should involve some sort of an analytic continuation.

In this conference, a very interesting suggestion for a non-perturbative definition of a realtime static potential in this spirit was put forward [24]. Motivated by the perturbative definition, the idea is to first measure a Wilson loop as a function of a Euclidean time coordinate, $\tau$; this can be illustrated as

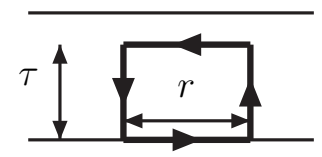

which observable we denote by $C_{E}(\tau, r) \equiv\left\langle\operatorname{Tr}\left[W_{E}(\tau, r)\right]\right\rangle$. Supposing that an analytic continuation can be carried out, $\tau \rightarrow$ it, a real-time potential could then be extracted from

$$
i \partial_{t} C_{E}(i t, r) \equiv V_{>}(t, r) C_{E}(i t, r) .
$$

Its static limit corresponds to $V_{>}(\infty, r)$.

It is appropriate to point out that the horizontal Wilson lines appearing in the definition of $C_{E}(\tau, r)$ are non-unique, as usual; however, to the extent that we can compensate for the specific choice by a normalization factor, $Z_{s}^{1 / 2}(r)$, so that the correlator reads $C_{E}(\tau, r)=Z_{s}(r) \tilde{C}_{E}(\tau, r)$, we see that $Z_{s}(r)$ drops out from the definition of $V_{>}(t, r)$. Such a normalization factor is indeed characteristic of the effective field theory framework that can be used for addressing the properties of heavy quarkonium [25]. 

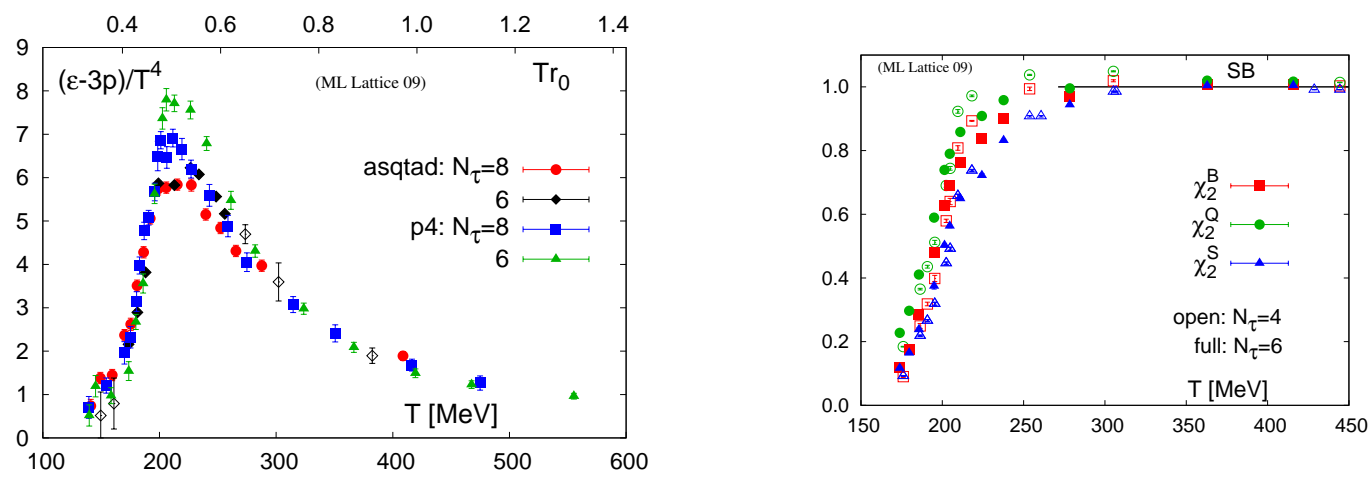

Figure 6: Left: the trace anomaly $(e-3 p) / T^{4}$ from ref. [26], with two families of fermion discretizations. Judging with bare eye, the results appear to converge towards a common continuum limit. Right: baryon number, electric charge, and strangeness susceptibilities $\left(\chi_{2}^{B}, \chi_{2}^{Q}, \chi_{2}^{S}\right.$, respectively), from ref. [27].

Now, according to ref. [24], the static limit $V_{>}(\infty, r)$ can indeed be extracted through a spectral analysis of the Euclidean correlator. More precisely, the data appear to indicate the presence of a spectral peak, whose position signals the average energy of the quark-antiquark system, $\operatorname{Re} V_{>}(\infty, r)$. The peak should have a finite width as well, $\operatorname{Im} V_{>}(\infty, r)$, being a signal of a Coulomb scattering/Landau damping induced "decoherence" of the quark-antiquark state [22], caused by collisions with the particles of the thermal medium.

To me, the idea of ref. [24] seems very interesting, and I am looking forward to further developments along these lines.

\section{Light quarks and gluons at high temperature}

I now move away from heavy quark related observables and discuss a number of recent developments related to light quarks and gluons.

\subsection{Basic thermodynamics with $N_{\mathrm{f}}=2+1, N_{\mathrm{c}}=3$}

Results for the trace of the energy-momentum tensor (like in fig. 1) and for various physical susceptibilities, from the large-scale simulations in refs. [26, 27], are shown in fig. 6. These results are supposed to be "physical", i.e. for (almost) realistic quark masses, and the lattice spacing dependence would appear to be regular as well. Improved staggered quarks were used.

It is fair to say, though, that it is not easy to judge the systematic uncertainties that may still be hidden in these results. For instance, in fig. 7 a comparison of the strangeness susceptibilities, as determined by the RBC-Bielefeld and by the Budapest-Wuppertal collaborations, is shown [28]. The infamous $30 \mathrm{MeV}$ temperature shift is clearly visible.

The good news is that the observable of fig. 7 is a nice one: it is directly physical, being related to the zero component of a conserved current, so that no renormalization ambiguities should appear. I encourage other groups to also primarily carry out comparisons with this quantity, rather than with more ultraviolet sensitive "auxiliary" observables, like the susceptibilities related to the Polyakov loop or the chiral condensate. 


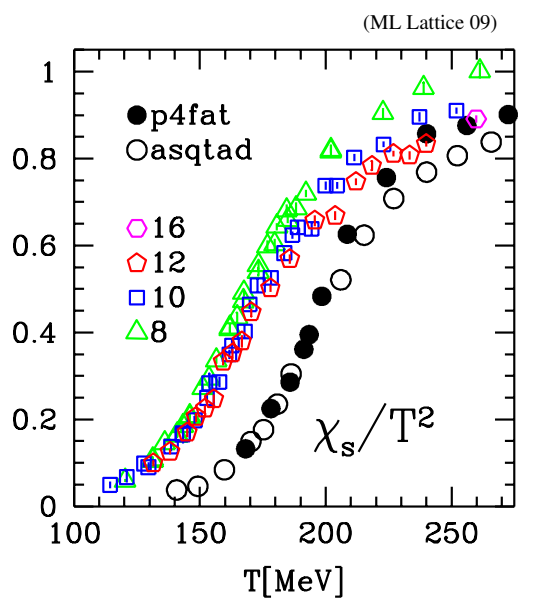

Figure 7: The strangeness susceptibilities from different collaborations, according to ref. [28].

As far as the reason for the discrepancy goes, I am certainly not qualified to offer any original insight. I do assume that "trivial" issues, like improper thermalization (see, e.g., ref. [30]), have been carefully excluded by all collaborations. As a "user" I do find it a pity, though, that none of the collaborations shows finite-volume scaling in their plots, even though the chiral limit is not far and the transition is very weakly of the first order; indeed figs. 6, 7 refer to a fixed box size $L$ in units of the temperature, $L T=3-4 .{ }^{1}$ On the side of discretization effects, it would be nice to overlay results from Wilson-like discretizations on fig. 7, particularly given the delicate role the chiral symmetry plays close to the transition point, and fortunately efforts in this direction appear to be under way $[31,32,33]$.

\subsection{New precision for $N_{\mathrm{f}}=0, N_{\mathrm{c}}=3$}

Recently, there have been new studies of the thermodynamics of pure SU(3) gauge theory. The great benefit of this simplified theory is that systematic errors can be brought better under control; therefore the theory offers an excellent test bench both for new lattice ideas, and for comparing the lattice data with various continuum computations. These two aspects are illustrated in fig. 8. On the left, a test is shown of the new approach of ref. [34], in which the lattice spacing is kept fixed and the temperature is varied through changing the number of points in the Euclidean time direction, $N_{\tau}$; this is theoretically more transparent than the standard approach where $N_{\tau}$ is kept fixed and temperature is varied through $\beta_{\mathrm{L}}$, implying a simultaneous variation of the lattice spacing. On the right, a very precise study of the entropy density at low temperatures is shown; the entropy density is a convenient observable in that it can be measured without any subtractions (because the entropy density of the vacuum state vanishes), through

$$
s=\frac{4}{3 T} Z_{2} \operatorname{Tr}\left[\mathbf{B}^{2}-\mathbf{E}^{2}\right],
$$

where $Z_{2}$ is a renormalization factor. It can be seen that the results are precise enough to allow for a stringent comparison with the contributions from various glueball spectra.

\footnotetext{
${ }^{1}$ Very recently scaling studies in the critical region have been reported in ref. [29].
} 

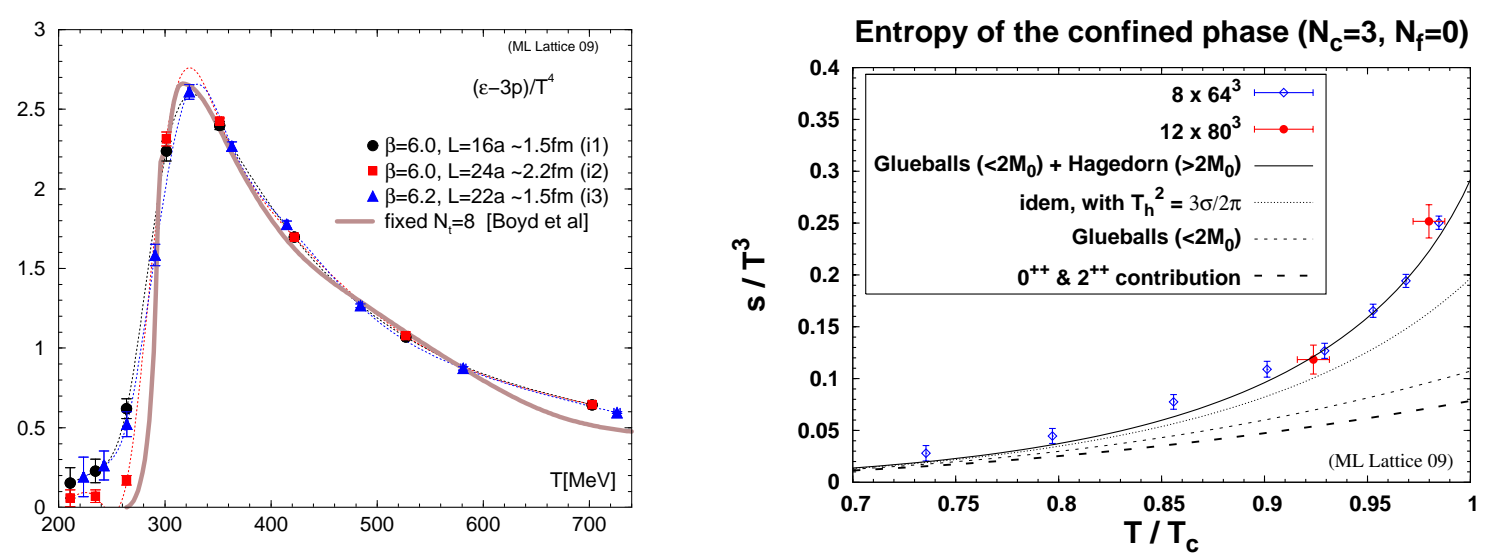

Figure 8: Left: the trace anomaly $(e-3 p) / T^{4}$ from ref. [34] for $N_{\mathrm{f}}=0$, in the so-called fixed-scale approach. Right: the entropy density from ref. [35] for $N_{\mathrm{f}}=0$, together with a comparison with various glueball resonance descriptions.

In my opinion, this kind of precision relooks at pure gauge theory are very welcome, and deserve to be pursued for many other observables as well.

\subsection{Another look at $N_{\mathrm{f}}=0, N_{\mathrm{c}}>3$}

Building on the previous section, it is also interesting to inspect pure gauge theories with $N_{\mathrm{c}}>$ 3. Recently two groups have come up with new results in this spirit (cf. fig. 9). In ref. [36], results have been presented for $N_{\mathrm{c}}=3,4,6$, with a focus on scale setting and continuum extrapolation $\left(N_{\tau}=6,8\right)$. Another work was presented in ref. [37] where, following the earlier work in ref. [38], results were presented for $N_{\mathrm{c}}=3,4,5,6,8$, for a fixed $N_{\tau}=5$.

A very intriguing trend can be extracted from the results of ref. [38] (fig. 9(right)). Indeed, it appears that for large $N_{\mathrm{c}}$, the functional form of the trace anomaly becomes much simpler than for $N_{\mathrm{c}}=3$ : once normalized to $N_{\mathrm{c}}^{2} T^{4}$, the result is basically zero for $T<T_{\mathrm{c}}$; displays a large jump (a first order transition) at $T=T_{\mathrm{c}}$; and decreases then monotonically for $T>T_{\mathrm{c}}$. This is a very simple pattern which suggests, and even calls for, a theoretical explanation; in fact, it can perhaps be speculated that in this limit the high-temperature phase is qualitatively purely perturbative.

A word of caution may be in order, though. In ref. [39], the latent heat (the discontinuity of the energy density or, for that matter, of the trace anomaly) was studied at various $N_{\mathrm{c}}$ for $N_{\tau}=$ $5,6,8$, and it was found that in general the results at $N_{\tau}=5 \mathrm{did}$ not fit well into the continuum extrapolation, particularly at large $N_{\mathrm{c}}$. Given that in ref. [37] only $N_{\tau}=5$ was considered, the results should probably be assigned a generous systematic error (as indeed elaborated upon in ref. [37]); in particular, it might be desirable to have additional $N_{\tau}$ 's to add confidence to the $N_{\mathrm{c}} \rightarrow \infty$ limit (for $N_{\mathrm{c}}=4,6$, the results of ref. [36] might be helpful in this exercise). In any case, I consider this to be a very interesting topic and worth further study.

\subsection{Finite-volume effects and screening masses}

In section 3.1, I already alluded to finite-volume effects. Recently, an interesting theoretical work appeared [40] in which the finite-volume effects in various thermodynamic observables 

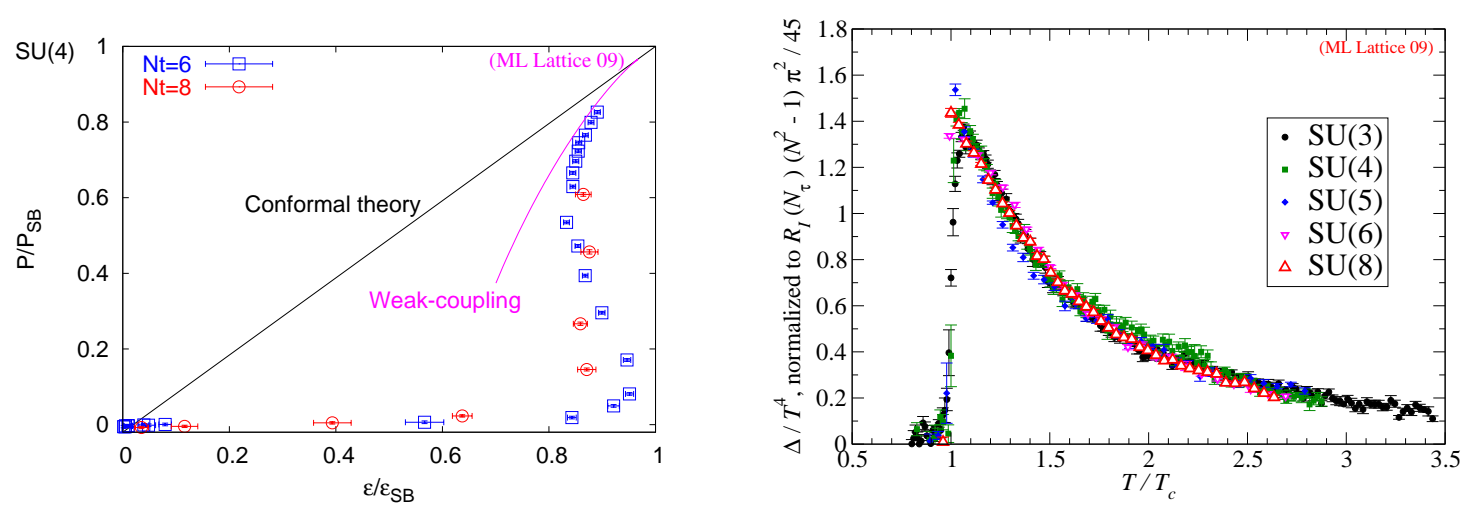

Figure 9: Left: pressure versus energy density for $\mathrm{SU}(4)$ gauge theory, compared with the conformal theory as well as with the weak-coupling prediction (from ref. [36]). Right: the trace anomaly $(e-3 p) / T^{4}$ for various $N_{\mathrm{c}}$, at a fixed $N_{\tau}=5$ (from ref. [37]).

were analyzed in some detail. For instance, for the entropy density, $s=(p+e) / T$, which can be measured according to eq. (3.1), the expression

$$
s(T, L)=s(T, \infty)-v \frac{e^{-m(T) L}}{2 \pi L}\left[1+\frac{3}{2} T \partial_{T}\right] m^{2}(T)+\mathscr{O}\left(e^{-\sqrt{2} m(T) L}\right)
$$

was given, with $m(T)$ denoting the lightest screening mass and $v$ its degeneracy. Similar formulae were also given for combinations like $e-3 p$ and $p$; the ones for $e-3 p$ would play a role in measurements such as those in figs. 6(left), 8(left), 9(right). The exponential dependence in eq. (3.2) is familiar, but it is interesting that the pre-exponential factor is also completely fixed in terms of known or measurable quantities.

In my opinion, the fact that finite-volume effects are determined by the lightest screening mass, as exemplified by eq. (3.2), underlines the usefulness of measuring the screening masses in the context of every lattice study. Indeed, though of no direct use for heavy ion experimentalists, the screening masses allow theorists to learn a lot about the dynamics of the system, and also to judge whether systematic errors related to finite-volume effects can be under control.

Examples of measurements of screening masses are shown in fig. 10. Among the qualitative observations that can be made from these plots are the following:

- For $T \gtrsim 1.5 T_{\mathrm{c}}$, the screening masses coupling dominantly to gluonic objects (i.e. flavour singlets) are the lightest ones. That this should happen is one of the predictions of the dimensionally reduced effective description of high-temperature QCD [43]; thus, we may assume that dimensional reduction could work qualitatively for $T \gtrsim 1.5 T_{\mathrm{c}}$ while it probably cannot capture all the relevant dynamics for $T \lesssim 1.5 T_{\mathrm{c}}$ if physical $N_{\mathrm{c}}$ and $N_{\mathrm{f}}$ are being used.

- It can be seen that cutoff effects in the mesonic screening mass become substantial at high temperature. It is interesting to compare this behaviour with that in fig. 7; much the same appears to be the case in the strangeness susceptibility.

- At low temperatures, on the other hand, cutoff effects in the mesonic screening mass appear to be moderate, while at the same time the screening mass itself becomes small, $m / T \sim$ 

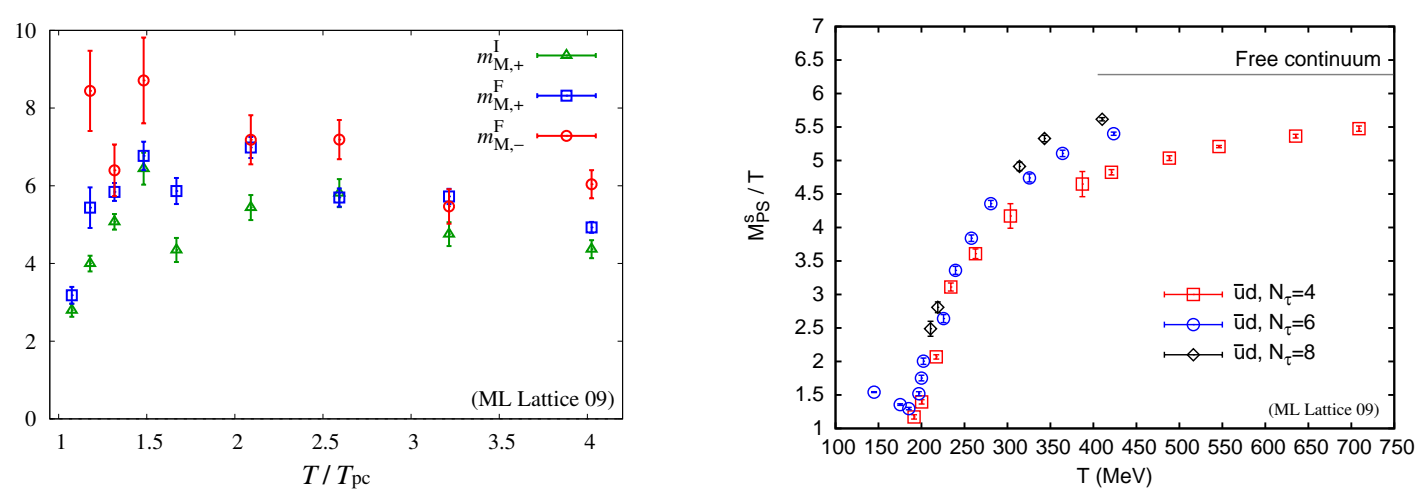

Figure 10: Left: the smallest screening masses extracted from glueball like operators, with $N_{\mathrm{f}}=2$ Wilson quarks in a $V=16^{3} \times 4$ volume (from ref. [41]). Right: the smallest screening mass extracted from a pseudoscalar mesonic operator, with $N_{\mathrm{f}}=2+1$ p4fat3 staggered quarks (from ref. [42]).

1 around $T \sim T_{\mathrm{c}}$, indicating the "vicinity" of a second order transition. This suggests, in accordance with the discussion around eq. (3.2), that finite-volume effects may be significant at low temperatures. For pure $\mathrm{SU}(3)$, this is clearly visible also in fig. 8(left).

Hopefully these points serve to illustrate that there is much to learn from screening masses, and encourage them to be adopted as a standard part of every finite temperature lattice study.

\subsection{Energy-momentum correlators and sum rules}

I wish to end with a somewhat amusing recent episode, related to the determination of transport coefficients. As was already discussed in section 2.2, extracting a transport coefficient from a conserved current is very difficult in general, because there may be a narrow transport peak in the corresponding spectral function, $\rho(\omega)$, around $\omega=0$, and one would need to extract the height of the peak. If, however, analytic information is available on the shape of the spectral function, then sum rules relate integrals over $\rho$ to various thermodynamic quantities, which are easier to measure.

Now, a particular quantity considered in this spirit is the standard hydrodynamic transport coefficient known as bulk viscosity. The sum rule states that

$$
S=\int_{0}^{\infty} \frac{\mathrm{d} \omega}{\omega}\left[\rho^{\text {bulk }}(\omega)-\rho_{T=0}^{\text {bulk }}(\omega)\right],
$$

where $S$ is a certain local thermodynamic observable. Setting aside the question (which I consider to be very difficult) of whether the functional form of $\rho^{\text {bulk }}(\omega)-\rho_{T=0}^{\text {bulk }}(\omega)$ can be reasonably modelled, there has even been confusion about how the left-hand side, $S$, looks like. In fact, two different answers have been proposed: one in refs. [44, 45], and more recently another one in ref. [46] (see ref. [47] for a related discussion). The two expressions are compared in fig. 11.

The reason for the difference is in some sense subtle, though eventually perhaps also simple to understand [46]. The older expression applies in Euclidean spacetime; it gives the value of a certain two-point function at a fixed Matsubara frequency $\omega_{n}=0$, extrapolated subsequently to vanishing spatial momentum. The other expression, in contrast, assumes first the limit of vanishing spatial momentum, while still keeping full information on all Matsubara frequencies $\omega_{n}$, in order to allow for an analytic continuation to Minkowskian frequency $\omega$. 


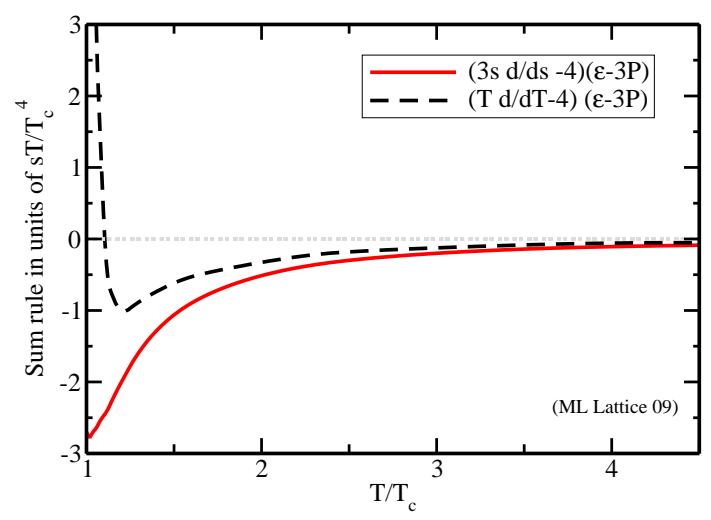

Figure 11: A comparison of two different quantities that have been thought to be relevant for the bulk viscosity sum rule, eq. (3.3) (from ref. [46]). The lattice data is for pure SU(3) and originates from ref. [48].

Clearly, fig. 11 demonstrates that it is important to have the correct left-hand side in the sum rule of eq. (3.3), if information concerning the right-hand side is to be extracted.

\section{Conclusions}

The main point which I wanted to illustrate in this talk is that, apart from the obvious need to continue numerical efforts in order to reach chiral, infinite-volume, and continuum limits with controlled systematic errors for physical QCD, there is also room and even need for various types of theoretical contributions to the understanding of strong interactions at high temperatures. This includes both analytic computations, examples of which were given in sections 2.2, 3.4 and 3.5, as well as numerical efforts in simplified theories, in which the systematic errors can be controlled more easily than in full QCD; examples were mentioned in sections $2.3,3.2,3.3$. Hopefully more groups will join also these physically motivated "low-cost" efforts in the coming years!

\section{Acknowledgements}

I wish to acknowledge useful discussions and correspondence with S. Borsányi, B. Bringoltz, F. Bruckmann, S. Datta, U. Heller, L. Levkova, Yu. Maezawa, M. Panero, A. Rothkopf and G. Schierholz, as well as financial support from the BMBF, under project Heavy Quarks as a Bridge between Heavy Ion Collisions and QCD.

\section{References}

[1] T. Hatsuda, PoS LAT2006 (2006) 010; U.M. Heller, PoS LAT2006 (2006) 011 [hep-lat / 0610114 ].

[2] G. Aarts, PoS LAT2007 (2007) 001 [0710 . 0739];

Z. Fodor, PoS LAT2007 (2007) 011 [0 711 . 0336];

F. Karsch, PoS LAT2007 (2007) 015 [0711 . 0661].

[3] C.E. DeTar, PoS LATTICE2008 (2008) 001 [0 811 . 242 9];

H.B. Meyer, PoS LATTICE2008 (2008) 017 [0 809 . 52 02]. 
[4] X.F. Meng, G. Li, Y. Chen, C. Liu, Y.B. Liu, J.P. Ma and J.B. Zhang, 0903.1991 ;

E. Bilgici et al, 0906.3957 ;

P.V. Buividovich, M.N. Chernodub, E.V. Luschevskaya and M.I. Polikarpov, 0907.0494 ;

B.H. Wellegehausen, A. Wipf and C. Wozar, 0907 . 1450;

D.K. Sinclair and J.B. Kogut, 0909.2019 ;

B.A. Berg, A. Bazavov and H. Wu, 0909.3077 ;

T. Saito, M.N. Chernodub, A. Nakamura and V.I. Zakharov, 0910.4828 ;

T. Blum, these proceedings;

F. Di Renzo, these proceedings;

Y. Kohno, these proceedings;

Y. Liu, these proceedings;

A. Nakamura, these proceedings.

[5] M. Srednicki, R. Watkins and K.A. Olive, Nucl. Phys. B 310 (1988) 693;

M. Hindmarsh and O. Philipsen, Phys. Rev. D 71 (2005) 087302 [hep-ph/0501232];

M. Laine and M. Shaposhnikov, JCAP 06 (2008) 031 [0 804 . 4 543 ].

[6] M. Laine and Y. Schröder, Phys. Rev. D 73 (2006) 085009 [hep-ph / 0603048 ].

[7] M. Cheng [RBC-Bielefeld Collaboration], PoS LAT2007 (2007) 173 [0710 . 4357].

[8] L. Levkova [MILC Collaboration], 0910.3006.

[9] M. Cacciari, P. Nason and R. Vogt, Phys. Rev. Lett. 95 (2005) 122001 [hep-ph / 0502203 ].

[10] B.I. Abelev et al. [STAR Collaboration], Phys. Rev. Lett. 98 (2007) 192301 [nucl-ex/0607012].

[11] A. Adare et al. [PHENIX Collaboration], Phys. Rev. Lett. 98 (2007) 172301 [nucl-ex/ 0611018 ].

[12] J. Casalderrey-Solana and D. Teaney, Phys. Rev. D 74 (2006) 085012 [hep-ph/ 0605199 ].

[13] S. Caron-Huot, M. Laine and G.D. Moore, JHEP 04 (2009) 053 [0 901 . 11 195].

[14] M. Laine, G.D. Moore, O. Philipsen and M. Tassler, JHEP 05 (2009) 014 [0 902 . 2856 ].

[15] J.B. Kogut and L. Susskind, Phys. Rev. D 11 (1975) 395;

D.Y. Grigoriev and V.A. Rubakov, Nucl. Phys. B 299 (1988) 67;

J. Ambjørn, T. Askgaard, H. Porter and M.E. Shaposhnikov, Nucl. Phys. B 353 (1991) 346;

D. Bödeker, Nucl. Phys. B 486 (1997) 500 [hep-th/9609170].

[16] S. Caron-Huot and G.D. Moore, Phys. Rev. Lett. 100 (2008) 052301 [0 708 . 4232];

S. Caron-Huot and G.D. Moore, JHEP 02 (2008) 081 [0 801 . 2173].

[17] Y. Akamatsu, T. Hatsuda and T. Hirano, Phys. Rev. C 79 (2009) 054907 [0809.14 99].

[18] S.S. Gubser, Nucl. Phys. B 790 (2008) 175 [hep-th/0612143].

[19] G. Aarts et al, Phys. Rev. Lett. 99 (2007) 022002 [hep-lat / 0703008 ];

H.B. Meyer, Phys. Rev. D 76 (2007) 101701 [0 0404 . 1801].

[20] L.D. McLerran and T. Toimela, Phys. Rev. D 31 (1985) 545;

H.A. Weldon, Phys. Rev. D 42 (1990) 2384;

C. Gale and J.I. Kapusta, Nucl. Phys. B 357 (1991) 65.

[21] T. Matsui and H. Satz, Phys. Lett. B 178 (1986) 416. 
[22] M. Laine, O. Philipsen, P. Romatschke and M. Tassler, JHEP 03 (2007) 054 [hep-ph / 0611300 ]; A. Beraudo, J.P. Blaizot and C. Ratti, Nucl. Phys. A 806 (2008) 312 [0712 . 4394]; M.A. Escobedo and J. Soto, Phys. Rev. A 78 (2008) 032520 [08 04.0691 ];

N. Brambilla, J. Ghiglieri, A. Vairo and P. Petreczky, Phys. Rev. D 78 (2008) 014017 [0804 . 0993 ].

[23] Y. Burnier, M. Laine and M. Vepsäläinen, JHEP 01 (2008) 043 [0711 . 1743].

[24] A. Rothkopf, T. Hatsuda and S. Sasaki, 0910.2321.

[25] N. Brambilla, A. Pineda, J. Soto and A. Vairo, Nucl. Phys. B 566 (2000) 275 [hep-ph/9907240].

[26] A. Bazavov et al. [hotQCD Collaboration], Phys. Rev. D 80 (2009) 014504 [0 903 . 4379 ].

[27] M. Cheng et al. [RBC-Bielefeld Collaboration], Phys. Rev. D 79 (2009) 074505 [0811. 1006 ].

[28] Y. Aoki et al, JHEP 06 (2009) 088 [0903 . 4155].

[29] S. Ejiri et al, 0909.5122.

[30] S. Schaefer, R. Sommer and F. Virotta, 0910.1465.

[31] E.M. Ilgenfritz et al, 0905.3112.

[32] K. Kanaya et al. [WHOT-QCD Collaboration], 0907 . 4205; K. Kanaya et al. [WHOT-QCD Collaboration], 0910.5284.

[33] V.G. Bornyakov et al, 0910.2392.

[34] T. Umeda et al [WHOT-QCD Collaboration], Phys. Rev. D 79 (2009) 051501 [0 809 . 2842].

[35] H.B. Meyer, Phys. Rev. D 80 (2009) 051502 [0905 . 4229].

[36] S. Datta and S. Gupta, 0909.5591 ;

S. Datta and S. Gupta, 0910.2889.

[37] M. Panero, 0907.3719.

[38] B. Bringoltz and M. Teper, Phys. Lett. B 628 (2005) 113 [hep-lat/ 0506034 ].

[39] B. Lucini, M. Teper and U. Wenger, JHEP 02 (2005) 033 [hep-lat/ 0502003 ].

[40] H.B. Meyer, JHEP 07 (2009) 059 [0905 . 1663].

[41] Yu. Maezawa et al. [WHOT-QCD Collaboration], PoS LATTICE2008 (2008) 194 [0811 . 0426 ].

[42] S. Mukherjee [RBC-Bielefeld Collaboration], Nucl. Phys. A 820 (2009) 283C [0810 . 2906].

[43] P. Ginsparg, Nucl. Phys. B 170 (1980) 388;

T. Appelquist and R.D. Pisarski, Phys. Rev. D 23 (1981) 2305.

[44] P.J. Ellis, J.I. Kapusta and H.B. Tang, Phys. Lett. B 443 (1998) 63 [nucl-th/9807071].

[45] D. Kharzeev and K. Tuchin, JHEP 09 (2008) 093 [0 705 . 4280].

[46] P. Romatschke and D.T. Son, Phys. Rev. D 80 (2009) 065021 [0 903 . 394 6].

[47] S. Caron-Huot, Phys. Rev. D 79 (2009) 125009 [0903 . 3958].

[48] G. Boyd et al, Nucl. Phys. B 469 (1996) 419 [hep-lat/9602007]. 